\title{
Novos registros de Eunotia Ehrenberg (Eunotiaceae-Bacillariophyta) para o Estado do Rio Grande do Sul e Brasil ${ }^{1}$
}

\author{
Aline Brugalli Bicca² e Lezilda Carvalho Torgan ${ }^{2,3}$
}

Recebido em 18/01/2008. Aceito em 27/08/2008

\begin{abstract}
RESUMO - (Novos registros de Eunotia Ehrenberg (Eunotiaceae-Bacillariophyta) para o Estado do Rio Grande do Sul e Brasil). O trabalho tem como objetivo apresentar as características morfológicas, e/ou estruturais e métricas de 12 espécies de Eunotia (E. batavica A. Berg, E. deficiens Metz., Lange-Bert \& García-Rodr., E. genuflexa Nörpel-Sch., E. hepaticola Lang-Bert. \& Wydrz., E. herzogii Krasske, E. mucophila (Lange-Bert., Nörpel-Sch. \& Alles) Lange-Bert., E. pileus Ehr., E. pirla Carter \& Flower, E. schwabei Krasske, E. subarcuatoides Alles, Nörpel-Sch. \& Lange-Bert., E. transfuga Metz. \& Lange-Bert. e E. yanomami Metz. \& Lange-Bert.) encontradas nas áreas da Lagoa do Casamento e dos Butiazais de Tapes, entre as coordenadas 30¹0'-3040'S e 50³0'-51³0'W, na Planície Costeira do Rio Grande do Sul. São incluídos comentários sobre a distribuição e os ambientes onde os táxons foram encontrados.
\end{abstract}

Palavras-chave: diatomáceas, distribuição, taxonomia

\begin{abstract}
New reports of Eunotia Ehrenberg (Eunotiaceae-Bacillariophyta) for Rio Grande do Sul State and Brazil). This paper presents the morphological and/or structural and mensurable characteristics of 12 Eunotia species (E. batavica A. Berg, E. deficiens Metz., Lange-Bert. \& García-Rodr., E. genuflexa Nörpel-Sch., E. hepaticola Lang-Bert. \& Wydrz., E. herzogii Krasske, E. mucophila (Lange-Bert., Nörpel-Sch. \& Alles) Lange-Bert., E. pileus Ehr., E. pirla Carter \& Flower, E. schwabei Krasske, E. subarcuatoides Alles, Nörpel-Sch. \& Lange-Bert., E. transfuga Metz. \& Lange-Bert. and E. yanomami Metz. \& Lange-Bert.) found in the Lagoa do Casamento and Butiazais de Tapes areas, between $30^{\circ} 10^{\prime}-30^{\circ} 40^{\prime} \mathrm{S}$ and $50^{\circ} 30^{\prime}-51^{\circ} 30^{\prime} \mathrm{W}$, on the Rio Grande do Sul State Coastal Plain. Comments on distribution and habitats where the taxons are found are included.
\end{abstract}

Key words: diatom, distribution, taxonomy

\section{Introdução}

Em 1999, no "Workshop de Avaliação de Ações Prioritárias para as Zonas Costeira e Marinha", as regiões da Lagoa do Casamento e dos Butiazais de Tapes foram indicadas como áreas insuficientemente conhecidas e prioritárias para estudo. Para atender a essa demanda desenvolveu-se, na Fundação Zoobotânica do Rio Grande do Sul, o projeto PRÓBIO, objetivando o inventário da biodiversidade desta região. O levantamento da microflora (Torgan et al. 2007) demonstrou que o gênero Eunotia se destacou pela riqueza de espécies, não tendo sido possível a identificação de todos os táxons, justificando assim, um estudo mais detalhado desses organismos. A presente investigação tem como objetivo apresentar as características morfológicas, e/ou estruturais e métricas de 12 espécies de Eunotia e sua distribuição na área de estudo. Trata-se de espécies pela primeira vez registradas e descritas para o Rio Grande do Sul e/ou Brasil.

Eunotia Ehrenberg é um gênero restrito à água doce e particularmente abundante no epifíton e metafíton em águas oligotróficas, ácidas e preferencialmente em ambientes lênticos (Round et al. 1990). Os organismos são na sua maioria cosmopolitas, ocorrendo, entretanto, formas que se limitam a regiões tropicais e subtropicais do globo (Metzeltin \& Lange-Bertalot 1998). Este gênero caracteriza-se por possuir valvas isopolares dorsiventrais, assimétricas em relação ao eixo apical e por apresentar rafe rudimentar e uma a duas rimoportulae por valva (Kociolek \& Spaulding 2003). O gênero está inserido em um grupo intermediário entre as arrafídeas primitivas e as rafídeas mais derivadas, as naviculóides (Novitski \& Kociolek 2005). Atualmente, são conhecidos cerca de 300 táxons específicos e infraespecíficos (Van Landingham 1969; Metzeltin \& Lange-Bertalot 1998; Krammer \& Lange-Bertalot 1991; Metzeltin et al. 2005).

\section{Material e métodos}

O estudo baseou-se em amostragens efetuadas em 21 estações georreferenciadas, localizadas nas áreas da

\footnotetext{
Parte da Dissertação de Mestrado da primeira Autora

Museu de Ciências Naturais, Fundação Zoobotânica do Rio Grande do Sul, C. Postal 1188, 90990-000 Porto Alegre, RS, Brasil

Autor para correspondência: lezilda.torgan@fzb.rs.gov.br
} 
Lagoa do Casamento e dos Butiazais de Tapes, margens leste e oeste da laguna dos Patos, na Planície Costeira do Rio Grande do Sul, entre as coordenadas $30^{\circ} 10^{\prime}$ $30^{\circ} 40^{\prime} \mathrm{S}$ e $50^{\circ} 30^{\prime}-51^{\circ} 30^{\prime} \mathrm{W}$ (Fig. 1), em clima subtropical. Diferentes ambientes aquáticos foram amostrados: lagoas interligadas, lagoas isoladas, banhados, canal e açude. As coletas foram realizadas em duas épocas do ano, na estação de outono (maio e junho), e de primavera (outubro a dezembro) de 2003.

Um total de 53 amostras foi obtido, as de plâncton foram efetuadas com a passagem de frasco na subsuperfície da água e as de metafíton através de espremido manual das raízes e caules de macrófitas aquáticas. As amostras de frasco foram fixadas com formaldeído (1:100) e as de espremido com solução de Transeau (1:1).

Na preparação do material, o conteúdo celular e a matéria orgânica da amostra foram removidos através de oxidação com água oxigenada $\left(\mathrm{H}_{2} \mathrm{O}_{2}\right)$ e dicromato de potássio $\left(\mathrm{K}_{2} \mathrm{Cr}_{2} \mathrm{O}_{7}\right)$ durante uma hora a uma temperatura de $100{ }^{\circ} \mathrm{C}$. Foram efetuadas várias lavagens com água destilada em centrífuga a $1.800 \mathrm{rpm}$ para a retirada do ácido. Um total de 80 lâminas foi preparado, com utilização da resina Naphrax (índice de refração de 1,74) como meio de inclusão. Parte do material foi oxidada também com ácido nítrico $\left(\mathrm{HNO}_{3}\right)$ e lavada para ser



Figura 1. Localização das áreas de estudo com as estações de amostragem na Planície Costeira do RS, Brasil. montada em lâminas de raios-X sobre stubs de alumínio. $\mathrm{O}$ material fixado e as lâminas permanentes encontram-se tombados no Herbário Prof. Dr. Alarich Schultz (HAS), da Fundação Zoobotânica do Rio Grande do Sul.

A análise das diatomáceas foi realizada ao microscópio óptico (MO) Zeiss Axioplan e ao microscópio eletrônico de varredura (MEV) JEOL JSM-6060. O enquadramento taxonômico das espécies seguiu o sistema de Round et al. (1990) e a identificação dos táxons foi baseada na obra original dos mesmos. A temperatura, $\mathrm{pH}$ e condutividade da água foram medidos in locus com aparelhos marca HACH modelos $50150 \mathrm{e}$ 50050, para demonstrar as condições ambientais em que as espécies foram encontradas.

\section{Resultados e discussão}

Eunotia batavica A. Berg, Bot. Notiser, pl. 5: 462, f. 186. 1939.

Fig. 2

Valvas com margem dorsal levemente convexa; margem ventral levemente côncava; extremidades cuneado-arredondadas; nódulos terminais próximos das extremidades; estrias transapicais paralelas a levemente radiadas nas extremidades. Compr. 58,5 $\mu \mathrm{m}$; larg. $7,5 \mu \mathrm{m}$; relação comprimento:largura $(\mathrm{R} \mathrm{c} / \mathrm{l}) 7,8$; estrias $13 \mathrm{em} 10 \mu \mathrm{m}$.

Material examinado: BRASIL. Rio Grande do Sul: Tapes, banhado com Sphagnum, estação 18, zona litorânea, 4/VI/2003, UTM 473385-6628689 (HAS 104221, lâm. 5831).

Dados ambientais: temp. $21^{\circ} \mathrm{C}$; pH 6,4 e cond. $36 \mu \mathrm{S} \mathrm{cm}{ }^{-1}$.

Eunotia deficiens Metz., Lange-Bert. \& García-Rodr., Iconogr. Diatomol. 15: 48, pl. 22, f. 5. 2005.

Fig. 3, 13

Valvas com margem dorsal convexa; margem ventral levemente côncava; extremidades valvares arredondadas, não destacadas do corpo valvar; nódulos terminais afastados das extremidades; estrias transapicais paralelas a levemente radiadas, mais concentradas nas extremidades; rimoportula presente em uma das extremidades da valva, centralizada no eixo apical, de posição obliqua à helictoglossa. Compr. 27,0-60,6 $\mu$ m; larg. 6-12,6 $\mu \mathrm{m}$; R c/l 4,5-6,5; estrias 9-12 em $10 \mu \mathrm{m}$; aréolas 45 em $10 \mu \mathrm{m}$.

Esta espécie foi anteriormente encontrada no lago Calado, Amazonas, e identificada como Eunotia luna Ehr. var. aequalis Hust. (Metzeltin \& Lange-Bertalot 1998). Posteriormente, no estudo de diatomáceas do Uruguai, Metzeltin et al (2005) reconheceram que se 
tratava de uma espécie nova, denominando-a E. deficiens. Esta difere de E. luna var. aequalis (Schmidt et al., pl. 286, fig. 35, 1874-1959) por não possuir a margem dorsal fortemente convexa.

Material examinado: BRASIL. Rio Grande do Sul: Capivari do Sul, banhado entre lagoa do Capivari e lagoa do Casamento, estação 1, zona litorânea, 5/V/2003, UTM 541830-6654326 (HAS 104092, lâm. 5809); Tapes, banhado com Sphagnum, estação 18, zona litorânea, 4/VI/2003, UTM 473385-6628689 (HAS 104221); Palmares do Sul, lagoa do Casamento, estação 4, zona litorânea, 19/XI/2003, UTM 542604-6650282 (HAS 104360, lâm. 5844).

Dados ambientais: temp. $21-24,4^{\circ} \mathrm{C}$; $\mathrm{pH}$ 6,4-6,9 e

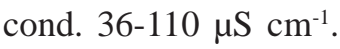

Eunotia genuflexa Nörpel-Sch., In Lange-Bertalot \& Metzeltin, Iconogr. Diatomol. 2: 50, pl. 9, f. 14-17. 1996.

Fig. 4, 14

Valvas lineares, levemente arqueadas; extremidades valvares arredondadas; nódulos terminais próximos das extremidades; estrias transapicais paralelas e eqüidistantes, interrompidas próximo às extremidades valvares; rimoportula presente em uma das extremidades da valva, levemente acima do eixo apical, de posição

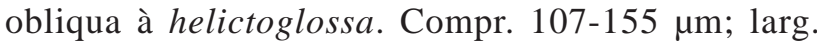
2,0-3,7 $\mu \mathrm{m}$; R c/l 35,5-53,0; estrias 18-20 em $10 \mu \mathrm{m}$; aréolas 50 em $10 \mu \mathrm{m}$.

Alguns exemplares possuem maior largura que o limite apresentado na circunscrição da espécie (1,5-2,6 $\mu \mathrm{m})$. Apesar dos indivíduos analisados possuírem sobreposição de medidas em relação à $E$. naegelii Mig. (Patrick \& Reimer 1966), esta se diferencia por apresentar as extremidades subcapitadas, voltadas para a margem dorsal da valva e menor relação comprimento/largura.

Material examinado: BRASIL. Rio Grande do Sul: Capivari do Sul, banhado entre lagoa do Capivari e lagoa do Casamento, estação 1, zona litorânea, 5/V/2003, UTM 541830-6654326 (HAS 104092, lâm. 5809); Tapes, banhado entre Dunas, estação 20, zona litorânea, 4/VI/2003, UTM 473603-6628803 (HAS 104234, lâm. 5815), 3/XII/2003, UTM 473603-6628803 (HAS 104449, lâm. 5863), banhado com Sphagnum, estação 18, zona litorânea, 4/VI/2003, UTM 473385-6628689 (HAS 104221, lâm. 5831).

Dados ambientais: temp. $17-26^{\circ} \mathrm{C}$; $\mathrm{pH} 5,4-6,7 \mathrm{e}$ cond. 32,3-110 $\mu \mathrm{S} \mathrm{cm}^{-1}$.

Eunotia hepaticola Lange-Bert. \& Wydr., Brenesia, 5556: 8-9, f. 1-9. 2001.

Fig. 5, 15
Valvas com margem dorsal convexa; margem ventral côncava; extremidades valvares dorsalmente capitadas, destacadas do corpo valvar; nódulos terminais próximos das extremidades; estrias transapicais paralelas, delicadas; rimoportula presente em uma das extremidades da valva, levemente acima do eixo apical, de posição obliqua à

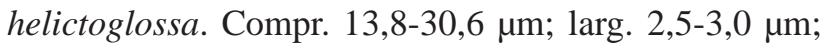
R c/1 4,6-10,9; estrias 20-24 em $10 \mu \mathrm{m}$; aréolas 50 em $10 \mu \mathrm{m}$.

Material examinado: BRASIL. Rio Grande do Sul: Tapes, banhado com Sphagnum, estação 18, zona litorânea, 3/XII/2003, UTM 473385-6628689 (HAS 104440, lâm. 5869); lagoa Redonda, estação 21, zona litorânea, 3/XII/2003, UTM 465503-6622735 (HAS 104455, lâm. 5867); lagoa Charutão, estação 14, zona pelágica, 2/XII/2003, UTM 465745-6623421 (HAS 104425, lâm. 5871), lagoa das Capivaras, estação 16, zona litorânea, 3/XII/2003, UTM 473595-6629067 (HAS 104435, lâm. 5846).

Dados ambientais: temp. $31,2-34^{\circ} \mathrm{C} ; \mathrm{pH} 4,3-6,3$ e cond. 13,3-42,1 $\mu \mathrm{S} \mathrm{cm} \mathrm{cm}^{-1}$.

Eunotia herzogii Krasske, Svensk bot. Tidskr. 42(4): 426, pl. 1, f. 20.1948.

Fig. 6

Valvas com margem dorsal convexa, apresentando quatro ondulações e a depressão central mais pronunciada; margem ventral reta; extremidades valvares dorsalmente capitadas, destacadas do corpo valvar, levemente voltadas para a margem ventral da valva; nódulos terminais distantes das extremidades; estrias transapicais paralelas a levemente radiadas em direção às extremidades. Compr. 32,4 $\mu \mathrm{m}$; larg. 4,2 $\mu \mathrm{m} ; \mathrm{R}$ c/1 7,7; estrias 10 em $10 \mu \mathrm{m}$.

Material examinado: BRASIL. Rio Grande do Sul: Tapes, lagoa Redonda, estação 21, zona litorânea, 3/XII/2003, UTM 465503-6622735 (HAS 104455, lâm. 5867).

Dados ambientais: temp. $31,2^{\circ} \mathrm{C} ; \mathrm{pH}$ 6,3 e cond. $13,3 \mu \mathrm{S} \mathrm{cm} \mathrm{cm}^{-1}$.

Eunotia mucophila (Lange-Bert., Nörpel-Sch. \& Alles) Lange-Bert., In Metzeltin et al., Iconogr. Diatomol. 15: 53. 2005.

Fig. 7, 16

Valvas fortemente arqueadas, com margem dorsal convexa; margem ventral côncava; extremidades valvares arredondadas, levemente destacadas do corpo valvar; nódulos terminais nas extremidades; terminação distal da rafe curvada em direção ao eixo apical; estrias transapicais paralelas e eqüidistantes. Compr.

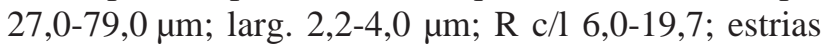
18-24 em $10 \mu \mathrm{m}$; aréolas 50 em $10 \mu \mathrm{m}$. 
Foi citada como E. bilunaris (Ehr.) Mills var. mисор hila Lange-Bert. \& Nörpel por Salomoni et al. (2006) no rio Gravataí, não podendo ser confirmada a identificação pela ausência de ilustração.

Material examinado: BRASIL. Rio Grande do Sul: Capivari do Sul, banhado entre lagoa do Capivari e lagoa do Casamento, estação 1, zona litorânea, 5/V/2003, UTM 541830-6654326 (HAS 104092, lâm. 5808); Palmares
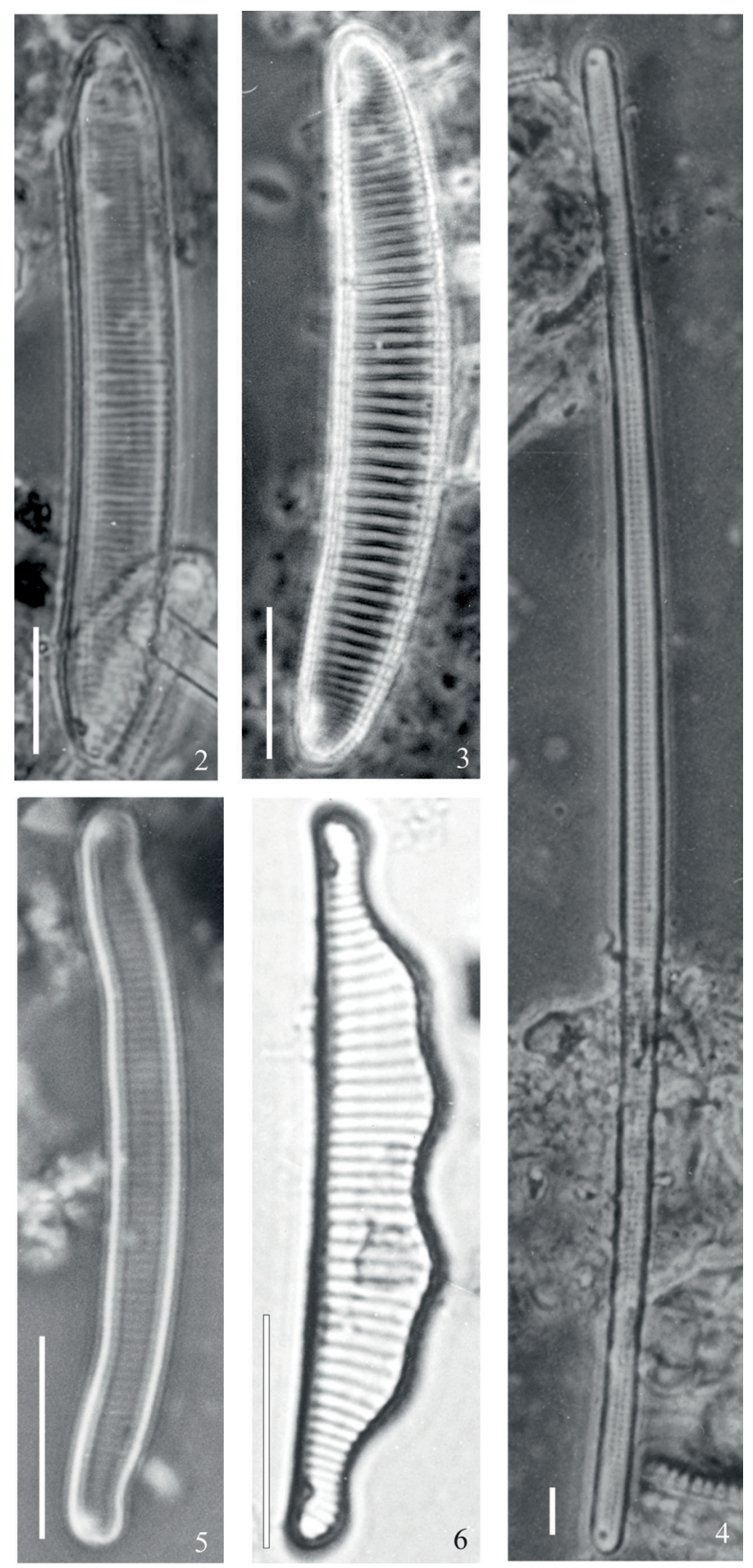

Figuras 2-6. Vista valvar das espécies de Eunotia em MO. 2. E. batavica A. Berg. 3. E. deficiens Metz., Lange-Bert. \& GarcíaRodr. 4. E. genuflexa Nörpel-Sch. 5. E. hepaticola Lange-Bert. \& Wydrz. 6. E. herzogii Krasske. Barras: $10 \mu \mathrm{m}$. do Sul, banhado Fazenda Rincão do Anastácio, estação 5, zona litorânea, 7/V/2003, UTM 530749-6639690 (HAS 104121, lâm. 5813 e 5814); Tapes, lagoinha entre Dunas, estação 19, zona litorânea, 4/VI/2003, UTM 473435-6628655, (HAS 104230, lâm. 5834A e 5955), banhado entre Dunas, estação 20, zona litorânea, 4/VI/2003, (HAS 104234, lâm. 5815 e 5818), 3/XII/2003, UTM 473603-6628803 (HAS 104449, lâm. 5863), lagoa das Capivaras, estação 16, zona litorânea, 3/XII/2003, UTM 473595-6629067 (HAS 104435), lagoa Redonda, estação 21, zona pelágica, 4/VI/2003, UTM 4655036622735 (HAS 104238, lâm. 5784), zona litorânea, 3/XII/2003, UTM 465503-6622735 (HAS 104455, lâm. 5867), lagoa do Charutão, estação 14, zona pelágica, 3/VI/2003, UTM 465745-6623421 (HAS 104196, lâm. 5816).

Dados ambientais: temp. $16,6-31,2^{\circ} \mathrm{C}$; $\mathrm{pH}$ 5,4-6,7 e cond. $12-110 \mu \mathrm{S} \mathrm{cm}^{-1}$.

Eunotia pileus Ehr., Abh. Dy. Akad. Wiss. Berl. Kl. 1841: 414, pl. II: f. I. 5 [Cayenne]. 1843.

Fig. 8

Valvas com margem dorsal convexa, apresentando duas ondulações; margem ventral côncava; extremidades valvares amplamente arredondadas, não destacadas do corpo valvar; nódulos terminais inconspícuos; estrias transapicais paralelas. Compr. 11,4 $\mu \mathrm{m}$; larg. 4,2 $\mu \mathrm{m}$; $\mathrm{R} \mathrm{c/l} \mathrm{2,7;} \mathrm{estrias} 10 \mathrm{em} 10 \mu \mathrm{m}$.

Material examinado: BRASIL. Rio Grande do Sul: Palmares do Sul, canal do Sangradouro, estação 10, zona litorânea, 19/XI/2003, UTM 532831-6631257 (HAS 104386, lâm. 5852).

Dados ambientais: temp. 21,7-21,9 ${ }^{\circ} \mathrm{C}$; $\mathrm{pH}$ 6,1 e cond. $13,7 \mu \mathrm{S} \mathrm{cm} \mathrm{cm}^{-1}$.

Eunotia pirla Carter \& Flower, Diatom Res. 3(1): 1-8. 1988.

Fig. 9

Valvas com margem dorsal convexa; margem ventral reta, intumescida no centro ou próximo das extremidades; extremidades valvares levemente voltadas para a margem ventral, destacadas do corpo valvar; nódulos terminais nas extremidades; estrias transapicais paralelas a radiadas em direção às extremidades. Compr.

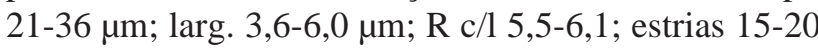
em $10 \mu \mathrm{m}$.

Material examinado: BRASIL. Rio Grande do Sul: Tapes, banhado com Sphagnum, estação 18, zona litorânea, 4/VI/2003, UTM 473385-6628689 (HAS 104221, lâm. 5831), 3/XII/2003, UTM 473385-6628689 (HAS 104440, lâm. 5869), lagoinha entre Dunas, estação 19, zona litorânea, 4/VI/2003, UTM 473435-6628655 (HAS 104230, lâm. 5955), lagoa Redonda, estação 21, 
zona pelágica, 4/VI/2003, UTM 465503-6622735 (HAS 104238 e 104240, lâm. 5784 e 5778), 3/XII/2003, UTM 465503-6622735 (HAS 104455, lâm. 5867).

Dados ambientais: temp. $17-32,5^{\circ} \mathrm{C} ; \mathrm{pH} 4,3-6,7$ e cond. 25,2-42,1 $\mu \mathrm{S} \mathrm{cm}^{-1}$.

Eunotia schwabei Krasske, Arch. f. Hydrobiol. 35: 366, pl. 10, f. 24-25. 1939.

Fig. 10

Valvas com margem dorsal fortemente convexa; margem ventral reta; extremidades valvares atenuadoarredondadas, destacadas do corpo valvar; nódulos terminais próximos das extremidades; estrias transapicais paralelas a levemente radiadas em direção às extremidades. Compr. 19,8-31,5 $\mu \mathrm{m}$; larg. 5,4-8,0 $\mu \mathrm{m}$; R c/1 3,6-3,9; estrias 12-13 em $10 \mu \mathrm{m}$.

Os exemplares estudados assemelham-se morfologicamente ao material-tipo, porém apresentam maior dimensão, pois os limites mencionados na descrição original são de 13-19 1/4m para o comprimento e de 4-5 $\mu \mathrm{m}$ para a largura.

Material examinado: BRASIL. Rio Grande do Sul: Capivari do Sul, banhado entre lagoa do Capivari e lagoa do Casamento, estação 1, zona litorânea, 5/V/2003, UTM 541719-6654246 (HAS 104096, lâm. 5811); Tapes, banhado com Sphagnum, estação 18, zona litorânea, 3/XII/2003, UTM 473385-6628689 (HAS 104440, lâm. $5869)$.

Dados ambientais: temp. $24,4-32,5{ }^{\circ} \mathrm{C}$; $\mathrm{pH}$ 4,3-6,6 e cond. $42,1-110 \mu \mathrm{S} \mathrm{\textrm {cm } ^ { - 1 }}$.

Eunotia subarcuatoides Alles, Nörpel-Sch. \& LangeBert., Nova Hedwigia 53: 188, pl. 4, f. 1-36. 1991.

Fig. 17, 18

Valvas com margem dorsal fortemente convexa; margem ventral levemente côncava; extremidades valvares atenuadas, não destacadas do corpo valvar; nódulos terminais próximos das extremidades; rafe curvada em direção ao eixo apical; estrias transapicais delicadas, paralelas a radiadas em direção às extremidades; rimoportula presente em cada extremidade da valva, localizadas no eixo apical e levemente abaixo, de posição obliqua à helictoglossa. Compr. 7,2-19,2 $\mu$; larg. 3,0-5,6 $\mu \mathrm{m}$; R c/1 2,4-3,7; estrias 16-20 em $10 \mu \mathrm{m}$; aréolas $50 \mathrm{em} 10 \mu \mathrm{m}$.

A espécie foi citada como E. vanheurckii var. intermedia (Krasske ex Hust.) Patr. por Laudares-Silva (1987), para o arroio do Faxinal, em Torres. Esta variedade, segundo Patrick-Reimer (p. 211, pl. 13, fig. $8,1966)$ é morfologicamente semelhante a $E$. subarcuatoides, diferindo, entretanto, pelo menor número de estrias (14 a $16 \mathrm{em} 10 \mu \mathrm{m})$.

Material examinado: BRASIL. Rio Grande do Sul:
Capivari do Sul, banhado entre lagoa do Capivari e lagoa do Casamento, estação 1, zona litorânea, 5/V/2003, UTM 541830-6654326 (HAS 104092, lâm. 5808), 27/X/2003, UTM 541830-6654326 (HAS 104341, lâm. 5771); ); Palmares do Sul, banhado Fazenda Rincão do Anastácio, estação 5, zona litorânea, 7/V/2003, UTM 5307496639690 (HAS 104121, lâm. 5814), lagoa dos Gateados Norte, estação 7, zona litorânea, 28/X/2003, UTM 531876-6628854 (HAS 104365, lâm. 5776); Mostardas, lagoa dos Gateados Sul, estação 12, zona pelágica, 31/X/2003, UTM 533600-6621912 (HAS 104403, lâm. 5874); Tapes, banhado com Sphagnum, estação 18, zona litorânea, 4/VI/2003, UTM 473385-6628689 (HAS 104221, lâm. 5831), 3/XII/2003, (HAS 104440, lâm. 5869), banhado entre Dunas, estação 20, zona litorânea, 3/XII/2003, UTM 473603-6628803 (HAS 104449, lâm. 5863).

Dados ambientais: temp. $16,6-32,5^{\circ} \mathrm{C}$; $\mathrm{pH} 4,3-6,7$ e cond. $36-202 \mu \mathrm{S} \mathrm{cm}^{-1}$.

Eunotia transfuga Metz. \& Lange-Bert. In LangeBertalot, Iconogr. Diatomol. 5: 84, pl. 9, f. 1-3. 1998. Fig. 11

Valvas lineares, levemente arqueadas, com margens dotadas de espinhos; extremidades valvares dilatadas, cuneado-arredondadas, destacadas do corpo valvar; nódulos terminais pequenos, nas extremidades; estrias transapicais paralelas a levemente radiadas em direção às extremidades. Compr. 142,5-199,0 $\mu \mathrm{m}$; larg. 7,8-9,0 $\mu \mathrm{m}$; R c/l 18,8-22,1; estrias 13-20 em $10 \mu \mathrm{m}$.

Citada como E. lineolata Hust. por Torgan \& Delani (1988) para o Complexo Banhado Grande, em Viamão e por Laudares-Silva (1987) no arroio do Faxinal, em Torres. Posteriormente, Metzeltin \& Lange-Bertalot (1998) transferiram as formas com extremidades dilatadas, cuneado-arredondadas de E. lineolata para E. transfuga.

Material examinado: BRASIL. Rio Grande do Sul: Tapes, lagoa das Capivaras, estação 16, zona litorânea, 3/XII/2003, UTM 473595-6629067 (HAS 104435, lâm. 5846), estação 17, zona pelágica, 4/VI/2003, UTM 473595-6629067 (HAS 104215, lâm. 5782), banhado com Sphagnum, estação 18, zona litorânea, 4/VI/2003, UTM 473385-6628689 (HAS 104221, lâm. 5831), lagoa Charutão, estação 14, zona pelágica, 2/XII/2003, UTM 465745-6623421 (HAS 104425, lâm. 5871); Palmares do Sul, canal do Sangradouro, estação 10, zona litorânea, 19/XI/2003, UTM 532831-6631257 (HAS 104386, lâm. 5852); banhado Fazenda Rincão do Anastácio, estação 5, zona litorânea, 7/V/2003, UTM 530749-6639690 (HAS 104121, lâm. 5814); Mostardas, lagoa dos Gateados Norte, estação 8, zona litorânea, 8/V/2003, UTM 532237-6625117 (HAS 104139, lâm. 5792), lagoa 
dos Gateados Sul, estação 12, zona pelágica, 31/X/2003, UTM 533600-6621912 (HAS 104403, lâm. 5874).

Dados ambientais: temp. $16-34{ }^{\circ} \mathrm{C}$; $\mathrm{pH} 5,6-6,7 \mathrm{e}$ cond. $12-362 \mu \mathrm{S} \mathrm{cm}^{-1}$.

Eunotia yanomami Metz. \& Lange-Bert., Iconogr.

Diatomol. 5: 86, pl. 34-37, 45, f. 2, 6, 63. 1998.

Fig. 12, 19-22

Valvas com margem dorsal convexa, apresentando duas ondulações; margem ventral côncava; extremidades valvares cuneado-arredondadas; nódulos terminais simples, junto às extremidades; rafe curvada em direção ao eixo apical; estrias transapicais nitidamente pontuadas, paralelas a radiadas em direção às extremidades. Compr.

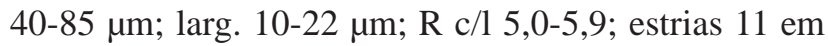
$10 \mu \mathrm{m}$; aréolas $24 \mathrm{em} 10 \mu \mathrm{m}$.

E. yanomami assemelha-se morfologicamente à E. zygodon Ehr., contudo, esta espécie apresenta nódulo trilobado (ver ilustração em Patrick \& Reimer, 1966, pl. 11, fig. 8) e maior número de aréolas nas estrias (Bicca et al., em preparação).

Material examinado: BRASIL. Rio Grande do Sul: Tapes, lagoinha entre Dunas, estação 19, zona litorânea, 4/VI/2003, UTM 473435-6628655, (HAS 104230, lâm. 5833), banhado entre Dunas, estação 20, zona litorânea, 3/XII/2003, UTM 473603-6628803 (HAS 104449, lâm. 5863), lagoa das Capivaras, estação 16, zona litorânea, 3/XII/2003, UTM 473595-6629067 (HAS 104435).

Dados ambientais: temp. $20-26,4{ }^{\circ} \mathrm{C} ; \mathrm{pH}$ 5,4-6,4 e cond. 12-70 $\mu \mathrm{S} \mathrm{cm}^{-1}$.

$\mathrm{O}$ gênero Eunotia encontra-se bem representado em águas continentais no estado do Rio Grande do Sul, com 96 táxons catalogados (Torgan et al. 1999). Dentre os 12 novos registros para o Estado, E. deficiens e E. hepaticola constituem-se também primeiras citações para o Brasil.

E. deficiens, E. genuflexa, E. mucophila, E. schwabei, E. subarcuatoides e E. transfuga tiveram ampla distribuição, estando presentes nas áreas da Lagoa do Casamento e dos Butiazais de Tapes. Dentre estas,
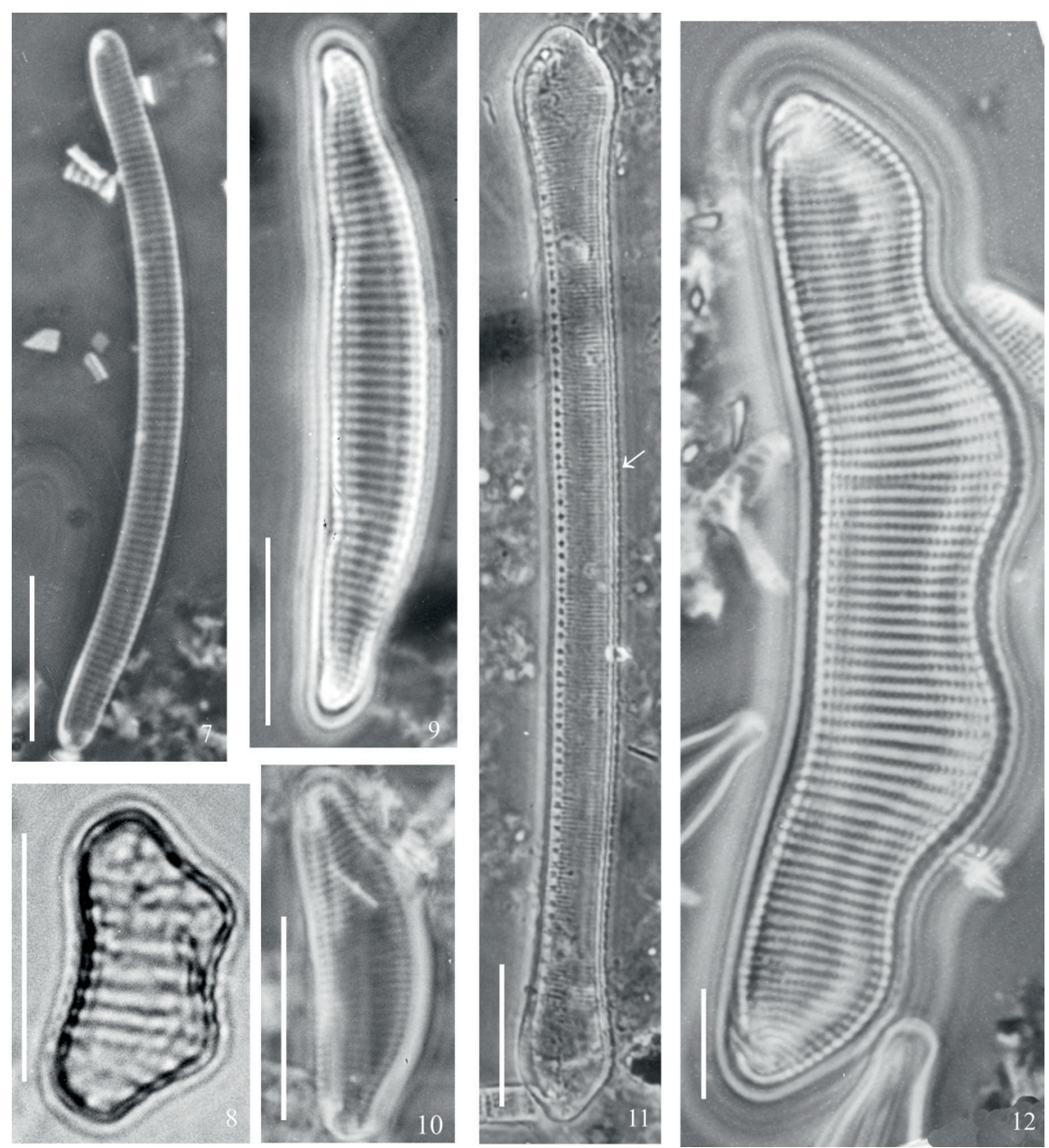

Figuras 7-12. Vista valvar das espécies de Eunotia em MO. 7. E. mucophila (Lange-Bert., Nörpel-Sch. \& Alles) Lange-Bert. 8. E. pileus Ehr. 9. E. pirla Carter \& Flower 10. E. schwabei Krasske. 11. E. transfuga Metz. \& Lange-Bert., mostrando os espinhos (seta). 12. E. yanomami Metz. \& Lange-Bert. Barras: $8=5 \mu \mathrm{m} ; 7,9-12=10 \mu \mathrm{m}$. 



Figuras 13-18. Vista valvar das espécies de Eunotia em MEV. 13. E. deficiens Metz., Lange-Bert. \& García-Rodr., vista interna mostrando a posição da rimoportula (seta). 14. E. genuflexa Nörpel-Sch., detalhe da extremidade interna mostrando a rimoportula (seta). 15. E. hepaticola Lange-Bert. \& Wydrz., vista interna mostrando a rimoportula (seta). 16. E. mucophila (Lange-Bert., Nörpel-Sch. \& Alles) Lange-Bert, detalhe da extremidade externa. 17-18. E. subarcuatoides Alles, Nörpel \& Lange-Bert., 17. Vista interna, 18. Vista externa. Barras: 13, 15, $17=2 \mu \mathrm{m} ; 14,16,18=1 \mu \mathrm{m}$.

E. subarcuatoides é considerada uma espécie cosmopolita (Krammer \& Lange-Bertalot 1991). E. genuflexa foi registrada na Finlândia em um lago rico em ácido húmico (Lange-Bertalot \& Metzeltin 1996), E. mucophila foi referenciada no Uruguai, em local não especificado (Metzeltin et al. 2005) e E. schwabei foi identificada por Krasske em lagos e queda d'água no Chile (Lange-Bertalot et al. 1996), sendo encontrada em charco de água sulfurosa na Argentina (Frenguelli 1942) e em ambiente com Sphagnum no Japão (Ando 1985).

E. hepaticola, E. herzogii, E. batavica, E. pirla e E. yanomami estiveram restritas à área dos Butiazais de Tapes, presentes em lagoas isoladas e banhados, em condições de temperatura mais elevada $\left(>20^{\circ} \mathrm{C}\right)$, baixo $\mathrm{pH}$ $(<6,4)$ e baixa condutividade $\left(<70 \mu \mathrm{S} \mathrm{cm}^{-1}\right)$. Tratam-se de espécies de origem tropical e pouco conhecidas.

E. hepaticola foi registrada até então somente para a Costa Rica, Alajuela, em um rio de altitude de água extremamente ácida (Wydrzycka \& Lange-Bertalot 2001). E. herzogii foi encontrada pela primeira vez no Brasil, mais especificamente em relva de Sphagnum, em local denominado Ilha da Mar, próximo a Santos, São
Paulo. E. yanomami foi mencionada para o lago Calado, na Amazônia Brasileira, e em rios nas Guianas (Metzeltin \& Lange-Bertalot 1998).

E. pirla foi encontrada em lagoas isoladas e banhado com Sphagnum, enquanto que E. batavica ocorreu somente no banhado com Sphagnum. São espécies também pouco conhecidas. E. pirla é originária de um lago ácido com Sphagnum no sul da Inglaterra (Carter $\&$ Flower 1988), enquanto que E. batavica tem origem duvidosa, sendo mencionada para o Rio Negro, sem a definição do país (Berg 1939).

Esta investigação vem demonstrar que parte das espécies encontradas na Planície Costeira do Rio Grande do Sul foi recentemente descoberta, enquanto que outras são pouco conhecidas, entretanto, estudos taxonômicos sobre o gênero fazem-se necessários, a fim de abranger outras Regiões Fisiográficas do Estado, onde há carência de informações.

\section{Agradecimentos}

À Coordenação de Auxílio à Pesquisa de Ensino 

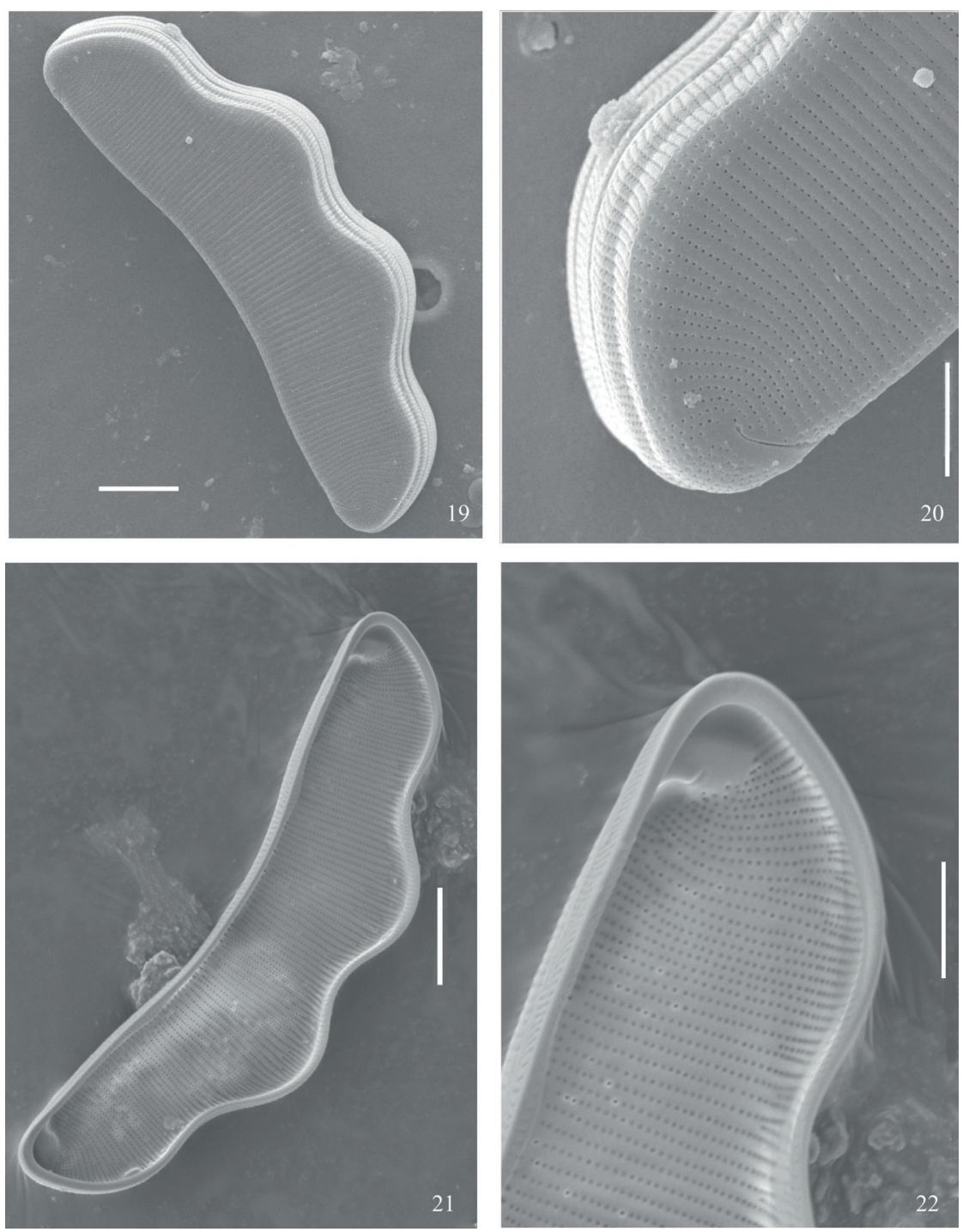

Figuras 19-22. Eunotia yanomami Metz. \& Lange-Bert. em MEV. 19. Vista valvar externa. 20. Detalhe da extremidade externa. 21. Vista valvar interna. 22. Detalhe da extremidade interna. Barras: $19,21=10 \mu \mathrm{m} ; 20,22=5 \mu \mathrm{m}$.

Superior (CAPES) e ao Conselho Nacional de Desenvolvimento Científico e Tecnológico (CNPq) pelas Bolsas de Mestrado e de Produtividade à Pesquisa à primeira e segunda autora, respectivamente. Ao Centro de Microscopia Eletrônica da UFRGS por disponibilizar o uso do microscópio eletrônico de varredura. Aos funcionários das Seções de Geoprocessamento e de Informática do Museu de Ciências Naturais/FZB pelo apoio técnico.

\section{Referências bibliográficas}

Ando, K. 1985. Moss diatoms in Japan. The Japanese Journal of Phycology 33: 143-148.

Berg, A. 1939. Some new species and forms of the Diatom genus Eunotia Ehrenberg 1873. Botaniska Notiser: 423-462.

Carter, J.R. \& Flower, R.J. 1988. A new species of Eunotia, E. pirla sp. Nov. from Woolmer Pond and acid pool in the southeast of England. Diatom Research 1: 1-8.

Ehrenberg, C.G. 1841-1843. Verbeitrung und Einfluss des mikroskopischen Lebens in Süd und Nord-Amerika. Bericht über die zur Bekanntmachung geeigneten Verhandlungen der Königlichen Preuss. Akademie der Wissenschaften zu Berlin.

Frenguelli, J. 1942. Diatomeas del Neuquén (Patagonia). Revista del Museo de La Plata (Nueva Serie) 3: 213-334.

Kociolek, J.P. \& Spaulding, S. 2003. Eunotioid and asymmetrical naviculoid diatoms. Pp. 655-668. In: J.D. Wehr \& R.G.F. Sheath. Freshwater algae of North America (Ecology and classification). London, Academic Press, Elsevier Science.

Krammer, K. \& Lange-Bertalot, H. 1991. Bacillariophyceae: Centrales, Fragilariaceae, Eunotiaceae. In: H. Ettl; J. Gerloff; H. Heyning \& D. Mollenhauer. Süsswasserflora von Mitteleuropa. Stuttgart, G. Fisher, v. 2, pt 3.

Lange-Bertalot, H.; Külbst, K.; Lauser, T.; Nörpel-Schempp, M. \& Willmann, M. 1996. Diatom Taxa Introduced by Georg Krasske - Documentation an Revision. In: H. Lange-Bertalot 
(ed.) Iconographia Diatomologica, annotated diatom micrographs. Stuttgart, Koeltz Scientific Books.

Lange-Bertalot, H. \& Metzeltin, D. 1996. Indicators of Oligotrophy. In: H. Lange-Bertalot (ed.). Iconographia Diatomologica, annotated diatom micrographs. Stuttgart, Koeltz Scientific Books.

Laudares-Silva, R. 1987. Estudo Taxonômico das Diatomáceas (Bacillariophyceae) coletadas no arroio do Faxinal (Sanga de Água Boa), Torres, Rio Grande do Sul, Brasil. Insula 17: 3-184.

Metzeltin, D. \& Lange-Bertalot, H. 1998. Tropische Diatoms of South America, 1. In: H. Lange-Bertalot (ed.). Iconographia Diatomologica, annotated diatom micrographs. Stuttgart, Koeltz Scientific Books.

Metzeltin, D.; Lange-Bertalot, H. \& García-Rodriguez, F. 2005. Diatoms of Uruguay. In: H. Lange-Bertalot (ed.). Iconographia Diatomologica, annotated diatom micrographs. Stuttgart, Koeltz Scientific Books.

Novitski, L. \& Kociolek, P. 2005. Preliminary light and scanning electron microscope observations of marine fossil Eunotia species with comments on the evolution of the genus Eunotia. Diatom Research 1: 137-143.

Patrick, R. \& Reimer, C.W. 1966. The diatoms of the United States exclusive of Alaska and Hawai. v.1. Philadelphia, Philadelphia Academy of Natural Sciences.

Round, F.E.; Crawford, R.M. \& Mann, D.G. 1990. The Diatoms: Biology and Morphology of the Genera. New York, Cambridge University Press.

Salomoni, S.E.; Rocha, O.; Callegaro, V.L. \& Lobo, E.A. 2006. Epilithic Diatoms as Indicators of Water Quality in the
Gravataí River, Rio Grande do Sul, Brazil. Hydrobiologia 559: 233-246.

Schmidt, A.; Fricke, F.; Heiden, H.; Müller, O. \& Hustedt, F. 18741959. Atlas der Diatomaceenkunde. Leipzig: Zentralantiquariat; Koenigstein/ Taunus, Otto Koeltz.

Torgan, L.C.; Becker, V. \& Prates, H.M. 1999. Checklist das diatomáceas (Bacillariophyceae) de ambientes de águas continentais e costeiros do estado do Rio Grande do Sul. Iheringia, Série Botânica 52: 89-144.

Torgan, L.C.; Alves-da-Silva, S.M.; Werner, V.R.; Rosa, Z.M.; Cardoso, L.S.; Rodrigues, S.C.; Dos Santos, C.B.; Palma, C.B.; Bicca, A.B.; Fortuna, J.R.; Weber, A.S. \& Martins, M.D. 2007. Ficoflora. Pp. 112-129. In: F.G. Becker; R.A. Ramos \& L.A. Azevedo (orgs.). Biodiversidade da região dos Butiazais de Tapes e da Lagoa do Casamento, Planície Costeira do Rio Grande do Sul. Brasília, MMA/SBF.

Torgan, L.C. \& Delani, O.M. 1988. Estudo taxonômico de diatomáceas (Bacillariophyceae) do "Complexo Banhado Grande", Rio Grande do Sul, Brasil: representantes do gênero Eunotia Ehrenberg. Iheringia, Série Botânica 38: 81-107.

Van Landingham, S.L. 1969. Catalogue of the fossil and recent genera and species of diatoms and their synonym. Pp. 1087-1756. Lehre, J. Cramer, v.3.

Wydrzycka, U. \& Lange-Bertalot, H. 2001. Las diatomeas (Bacillariophyceae) acidófilas del río Agrio y sítios vinculados com su cuenca, vólcan Poás, Costa Rica. Brenesia 55-56: $1-68$. 